\title{
Functionalised particles using dry powder coating in pharmaceutical drug delivery: promises and challenges
}

Eman Z. Dahmash, Afzal R. Mohammed*

Aston Pharmacy School, Aston University, Birmingham UK

* Corresponding Author:

Aston Pharmacy School, Aston University, Aston Triangle, Birmingham B4 7ET UK.

Phone: +44 1212044183 .

Email address: a.u.r.mohammed @aston.ac.uk 


\begin{abstract}
Introduction: Production of functionalised particles using dry powder coating is a one-step environmentally friendly process that paves the road for the development of particles with targeted properties and diverse functionalities.
\end{abstract}

Areas covered: Applying the first principles in physical science for powders, fine guest particles can be homogeneously dispersed over the surface of larger carrier particles to develop functionalised particles. Multiple functionalities can be modified including: flowability, dispersibility, fluidisation, homogeneity, content uniformity and dissolution profile. The current publication seeks to evaluate the different technologies developed over the last three decades to produce functionalised particles along with outlining their advantages, limitations and applications. The review aims to understand the fundamental principles governing the under pinning principle and mode of equipment operation and discusses in detail the resultant functionalities and their applications.

Expert opinion: Due to the ease in product development and manufacturability, dry particle coating is a promising and cost effective technique to produce particles with targeted functionalities. The process is underutilised within the pharmaceutical industry owing to the gap in this domain pertinent to the lack of availability of suitable devices that can process material without particle attrition, heat generation and contamination. With the growing demand in pharmaceutical industry for solvent free manufacturing techniques and continuous processing, extensive efforts are needed to develop suitable equipment that can deliver the plethora of diverse applications that can be investigated for drug formulation and delivery.

Keywords: Carrier, dry-powder coating, functionalised particles, guest, hybrid mixing, shear force 


\section{Article highlights}

- Blending process is critical in pharmaceutical manufacturing with non-uniform blending resulting in variation in content of the active pharmaceutical ingredient (API) and rejection of the final dosage from.

- Cohesiveness is attributed to strong interparticulate bond between particles resulting in poor flowability.

- Dry powder coating is a process where fine cohesive guest particles are adsorbed on to the surface of large carrier particles due to forces that are stronger than the weight of the guest particles.

- Dry coating is a one-step, environmentally friendly process requiring no solvents, drying or granulation.

- Several devices which can be classified as high impact or soft coating equipment have been developed to produce functionalised particles using dry coating.

- Various applications have been studied including enhancement of flowability, dispersibility, homogeneity and dissolution of water insoluble actives.

This box summarises key points contained in the article 


\section{Introduction}

\subsection{The science of powder blending}

Dry powder blending is a topic of significant interest due to its applications in multiple industries including the food industry, material processing and the pharmaceutical sectors $[1$, 2]. Following the Barr Laboratory court case in 1993 the process of powder blending has received substantial recognition $[3,4]$. In a survey conducted by BUWG of PQRI (Blend Uniformity Working Group of the Product Quality Research Institute), the use of direct compression as the main method for dosage form development accounts for $67 \%$ while wet granulation is used in $32 \%$ of the dosage forms followed by $1 \%$ for dry granulation [5]. Therefore, efficient and reproducible blending process is critical to manufacturing as deficiencies can lead to a non-homogenous mixture resulting in variation in the content of the active pharmaceutical ingredient (API) and rejection of the final dosage form [3]. The variation in content uniformity is caused by several factors including: poor flowability of components, segregation of the powder blend resulting from differences in particle size of the individual components, inappropriate blending process or blending equipment, agglomeration of components due to the presence of fine particles or moisture content and softening of components with low melting point $[2,6-8]$. Blending of materials to produce a state of homogeneity is an energy consuming process that results in a randomly distributed mixture where the efficiency of blending is largely dependent on various parameters pertinent to particle size, shape, size distribution, density, hardness and cohesivity. The type of mixing device and processing parameters are additional contributing factors and include speed, processing time and load $[9,10]$.

The different types of pharmaceutical materials that are routinely used can be broadly classified into two classes. The first is the non-cohesive free flowing material that 
demonstrates excellent flowability but handling is an issue due to segregation tendency that leads to a non-homogenous mixture. In order to obtain a homogenous mixture, particles should be within the same size, density and shape. The second class is the cohesive materials that tend to aggregate causing flowability disputes $[11,12]$. In the pharmaceutical industry the majority of APIs are present as cohesive powders. With solubility concerns, where the mainstream of APIs is within BCS class II (i.e., poor solubility but high permeability) the need for large surface area to promote dissolution is favoured [13, 14]. Therefore, selection of a mixing technique where the cohesiveness is minimised while the surface area is maximised is crucial.

\subsection{Origin of powder cohesiveness}

Cohesiveness is based on the high interparticulate force between micron or sub-micron particles that exceeds their weight [15]. Cohesive powder flows in coherent blocks that are larger than their primary particle size. The ratio between the interparticulate bonds to the particle weight in a given fixed volume is known as the cohesive granular bond number. This number tends to be very high for cohesive powder [15]. The interparticulate force is inversely proportional to the radius of the particle and as a result a reduction in the particle size will produce an increase in the cohesive forces and vice versa $[15,16]$. Materials are classified into four categories according to their ability to fluidise based on particle size and density (termed Geldart Classification). Type-A material represents aeratable, or homogeneously fluidised material, type-B is sand like material, type-C represents non-fluidisable cohesive material with mean particle size below $50 \mu \mathrm{m}$ and finally, class- D is a group represented by particles with size $>600 \mu \mathrm{m}$ and high density (spoutable material) thus requiring high energy to fluidise $[15,17,18]$.

Interparticulate bonding could be attributed to various forces such as surface tension that is caused by the liquid contained within the powder bed, electrostatic forces and van der Waals 
forces. Van der Waals forces are the main forces responsible for cohesion when the powder is dry $[10,12,19]$. For surface tension, liquid droplets form bridges and affect the curvature of the liquid gas interface leading to cohesion. Usually, when small amount of liquid is adsorbed to the surface of the powder particles, it results in changes in its surface energy and cohesive properties. If the water content is increased, it forms liquid bridges and leads to capillary forces which are established due to condensed moisture on the surface of the particles [12] [15]. Electrostatic forces are predetermined by charges present on the particles. Particles of different materials interchange charges during contact (e.g., triboelectricity), (those charges will be trapped in non-conducting particles) resulting in particle charge [15]. The interparticulate attraction force between particles with electrostatic charges is relatively high since the distance between particles in the powder bed is very small. If the electrostatic force is in the magnitude of the weight of the particles, or greater, the resultant cohesive forces prevent particle flow $[12,16]$. Generally, for dry fine neutral material, the electrostatic force is negligible compared to van der Waals forces. As for van der Waals forces, it is known that particles attract each other even in the neutral state as the instantaneous positions of the electrons around the nuclear protons produces a finite electric dipole that creates an electric field which polarizes a nearby molecule inducing a dipole which results in the attractive force between particles [15].

\subsection{Developing functionalised particles using dry powder coating}

When blending two components in a binary mixture, individual particles are redistributed throughout the system through diffusion and convection currents as well as shear and impaction forces producing a randomly distributed mix such that samples taken from the blends will produce uniform homogenous mixture. However, if the mixture contains a cohesive component, it results in the formation of agglomerates within the system or alternatively a difference in particle size between the two components leads to segregation 
producing a non-homogenous mixture [19]. In 1975, Hersey used the term "ordered mixing" to describe the cohesive powder blending process when fine particles are adhered on to the surface of coarse particles and termed it an "interactive mixture" [19-22]. On the other hand, hybrid mixing or dry powder coating was introduced later and indicates a strong adhesion of the fine (guest) particles on to the surface of coarse (carrier) particles [13]. Ishizaka and coworkers (1989) defined hybrid mixing in the context of particulate design to augment the quality of the material. In other words, the initial stage of hybrid mixing is the interactive mixture that upon application of additional stronger shear and compaction mechanical forces leads to an enhanced configuration depositing and fixing the guest particles on to the surface of the carrier [19].

One of the distinct advantages of developing hybrid mixtures is to overcome issues associated with segregation of particles due to differences in particle size. If the difference in size between the guest and the carrier is of at least one to two orders of magnitude, hybrid coating will ensure deposition and subsequent adhesion of the finer particles on to the surface of the coarser particles thereby overcoming disproportionate distribution of the particles in a mixture. The adhesion is due to the force that is greater than the weight of guest particles producing the ordered mixing or interactive mixture $[10,20,21,23,24]$. The fine guest particles are loosely (reversible process) and usually temporarily adsorbed to the carrier [13, 20, 21]. Hybrid mixing or dry particle coating is used to describe a special type of ordered /interactive powder mixing. With the aid of tailored blending devices that produce strong shear and impaction energy, guest particles are brought into close contact with the carrier causing it to be attached or even embedded onto the carrier's surface resulting in strong attraction that resembles coating $[10,13,23,25,26]$.

The aim of the present work is to provide an overview on the production of functionalised particles using dry powder coating. After a general introduction into the science behind the 
development of functionalised particles the main technologies involved in its production will be discussed in detail with special emphasis on applications in the pharmaceutical drug delivery sector.

\section{Science behind the formation of functionalised particles using dry powder coating}

During dry powder coating, the forces between the guest and the carrier could be both physical as well as chemical in nature [20, 23]. Production of functionalised particles through dry coating process is broadly carried out in three stages: initial stage involves deagglomeration of the cohesive guest particles, followed by the second phase where the fine guest particles adhere to the carrier surface (interactive mixtures) and finally the last stage that results in the redistribution of the guest within the carrier to reach a uniform spatial distribution of the guest particles. Different terminologies have been employed to describe this blending process and include hybrid mixing [20], composite powder formation [27], mechanical treatment, encapsulation [28], microfabrication [19], dry powder coating, engineered particles [23], mechanical dry coating [29] and surface adsorption [22].

Dry powder coating is divided into different categories according to the degree of coverage starting from the interactive ordered mix which comprises of loose surface coating to partial coating (discrete), followed by film formation and encapsulation (continuous coat) (Figure1) $[23,26,30,31]$. When the force of attraction between the fine guest particles and the carrier particles is stronger than the weight of the guest particles, robust functionalised particles that can withstand handling can be produced [32]. 


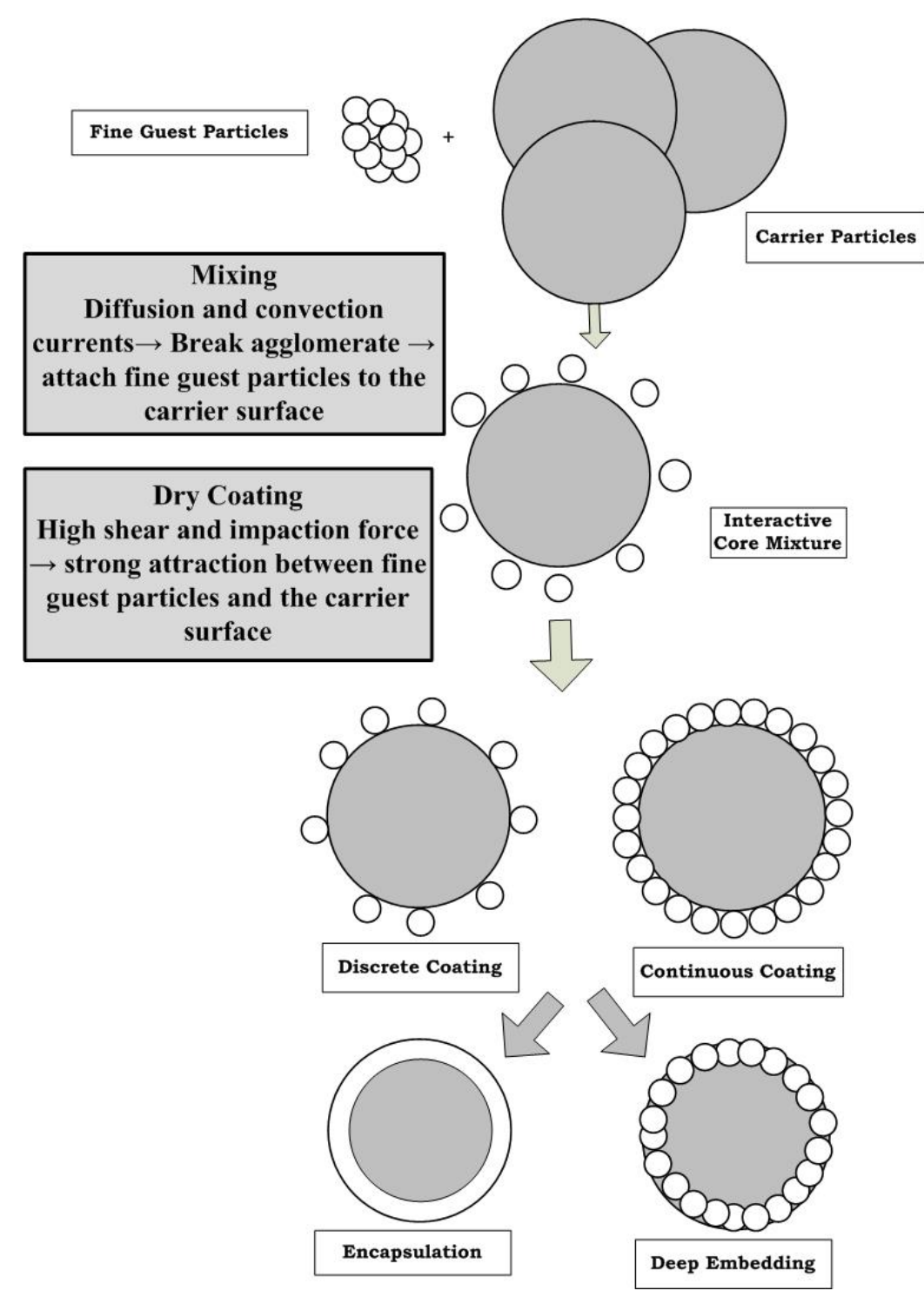

Figure 1. Schematic illustration showing interactive mixture and all types of dry coated particles. The inner part is the coarse carrier particle and the outer is particles of the cohesive fine guest particles. Adapted from [31].

In 1989, Ishizaka and colleagues [20] prepared dry powder coated and interactive mixtures and studied their stability over time using scanning electron micrographs (SEM). The results demonstrated that functionalised particles produced by the two methods showed similar morphology initially. However, upon conducting the stability tests over a period of 105 days, the dry coated particles retained their morphological features whereas interactive mixtures showed particle separation and crystal growth confirming the need for particle adsorption for long term stability of the powder mixture. The type and extent of dry powder coating is 
dependent on the physical properties of the guest material including its particle size, shape, roughness and melting point. Surface modifications and appearance of the functionalised particles range between irregular surface of crushed and micronized particles to smooth surface coat (e.g., oxyphenbutazone). Complete surface coverage can be attained when the mechanical energy introduced through dry powder coating device is sufficient to break the crystal lattice/cohesive agglomerates of the guest material and provides centripetal acceleration and energy for particle collision (between carrier and guest) resulting in permanent adsorption of the fine guest particles on to the surface of the carrier [20].

Honda and colleagues [19] reported the various factors that control effective hybrid mixing including the carrier/guest ratio, particle size of the two components and the force exerted during mixing. Additionally, different crystal forms of the API can result in different affinities to the carrier [11]. The equation below represents the role of number and size of the guest and carrier particles that need to be considered in the process of formation of hybrid blends.

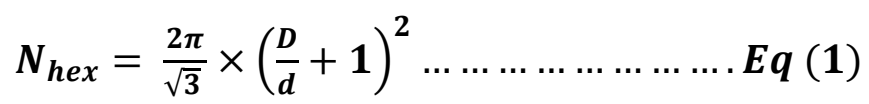

Where $(D)$ is the diameter of the carrier particles while $(d)$ is diameter of the fine guest particle. $(\mathrm{N})$ is the number of guest particles in contact with the surface of the carrier (hexagonally shaped). From the equation, it can be concluded that the ratio (D/d) is the predominant factor affecting the degree of packing [31]. It was reported by Alonso and coworkers that the guest particles are not likely to cover the surface of the carrier uniformly but rather will adhere randomly over the surface of the carrier leading to a total number being a fraction of $\mathrm{N}_{\text {hex }}$. However, upon introduction of strong shear and compaction force into the blend, the de-agglomerated fines will come into contact with the carrier leading to the 
transfer of guest molecules either between different carrier particles or the parent carrier particle resulting in a more uniform arrangement over the surface of the carrier $[30,31,33]$.

The relationship between guest to carrier size had further been explored within the context of uniform coating $[32,34]$. Therefore, it was proposed for dry powder that the weight coverage is calculated according to equations 2 and 3[32].

$$
\begin{gathered}
\boldsymbol{W t} \%=\frac{\left(\boldsymbol{N d ^ { 3 } \boldsymbol { \rho } _ { d } )}\right.}{\left(D^{3} \boldsymbol{\rho}_{D}\right)+\left(\boldsymbol{N} \boldsymbol{d}^{3} \boldsymbol{\rho}_{d}\right)} \times \mathbf{1 0 0} \% \ldots \ldots \ldots \ldots \ldots \ldots \boldsymbol{E q} \mathbf{2} \\
\boldsymbol{N}=\frac{\mathbf{4}(\boldsymbol{D}+\boldsymbol{d})^{2}}{d^{2}} \ldots \ldots \ldots \ldots \ldots \ldots \ldots \ldots \boldsymbol{E q} \mathbf{3}
\end{gathered}
$$

Where $(D)$ is the diameter of carrier particle, $(d)$ is the diameter of the guest particle, $\left(\rho_{\mathrm{d}}\right)$ is the density of the guest particle and $\left(\rho_{D}\right)$ is the density of carrier particle and $(\mathrm{N})$ is the number of guest particles that were coated on the surface of the carrier. It can be concluded that surface coverage of guest particles is crucial and is dictated by particle characteristics including size and density [32].

Zhou and colleagues $[35,36]$ investigated the effect of carrier particle size on flow properties using lactose as a model carrier dry coated with magnesium stearate as a guest material using mechanofusion ${ }^{\circledR}$. The results indicated that the carrier particle size has a crucial effect on improving the flowability. Tapped density increased with the increase of particle size and upon surface modification the increase in bulk and tapped densities was more pronounced within the smaller particle size range i.e., $4-20 \mu \mathrm{m}$ whereas for particles with larger size there was minimal change to both bulk and tapped densities. This difference in behaviour was attributed to the increase in void volume with larger particles compared to fine and the study concluded that the wider the polydispersity, the less is the increase in bulk and tapped densities since polydisperse particles pack more efficiently due to smaller particles filling in the interstices created by the large particles. Moreover, the carrier's particle shape plays an 
essential role in the degree and the efficiency of coating [29]. Using mechanofusion ${ }^{\circledR}$, dry coating was carried out and results revealed that the coating produced an increase in bulk and tapped density as well as reduction in shear stress [29].

To explain the adhesion behaviour between rough surfaces Xie and colleagues [37] carried out a study where it was presumed that the roughness of the particle surface (asperity) could be visualised as a smaller particle being attached to the surface of a smooth particle. The results demonstrated that van der Waal forces between particles is reduced when the asperities are less than $10 \mathrm{~nm}$ whereas they dominate when the size is more than $0.1 \mu \mathrm{m}$. Furthermore, Das and co-workers reported that the measured adhesion force between particles is less than the calculated one and this is due to the asperities on the surface that reduce surface contact area [38]. This principle provides the basis for functionalised particles produced using dry coating with reduced cohesiveness.

\section{Advantages of dry powder coating technique}

Dry powder coating offers several advantages as a blending technique particularly for direct compression of fine APIs by providing homogenous free flowing mixture [23, 39, 40]. Functionalised particles produced using dry powder coating have modified properties including enhanced flowability, solubility and wettability in addition to improvement in optical, catalytic and electrical properties. Enhancement of colour, taste or flavour has also been reported $[10,13,19,23,25]$. The process is cost effective where no additional materials are needed as with wet granulation. It is a green and environmentally friendly process requiring no solvent and is free of any process by-products. Furthermore, the process involves energy saving since there is no drying process $[13,19,20,23,41]$. Some devices where milling is concurrently carried out with coating, there is a reduction in the risk of 
contamination as there is no milling medium required [42]. The process is single step and easy to scale up $[26,29]$.

\section{Principles under pinning dry powder coating to formulate functionalised particle}

Various methods have been proposed for the development of interactive mixtures. The most commonly used technique is based on placing the fine powdered guest material between two layers of coarse carrier in a glass vial followed by vigorous hand shaking for 5 minutes [43]. Mechanical tumbling is another method for producing interactive mixtures. Additionally, turbula mixer, $\mathrm{V}$ blender and cube mixer have also been used to prepare interactive mixtures $[22,43]$. On the other hand, various devices based on either high or mild shear forces have been developed for dry powder coating and discussed in literature $[22,23,26]$.

\subsection{High impact devices}

The first device used for dry powder coating was Angmill ${ }^{\circledR}$, a grinding equipment that produced ultrafine particles through strong mechanical force and was built by a Japanese research group. This was later (1980s) modified to produce strong shear force from very high centrifugal and compression forces leading to surface fusion which laid the foundation for the concept of mechanofusion ${ }^{\circledR}$ [44]. Mechanofusion ${ }^{\circledR}$ device consists of rotating cylindrical processing vessel with a fixed scraper blade and inner-piece. The space between the innerpiece and the walls is adjustable (ranges between $2-5 \mathrm{~mm}$ ), whereas the space between the scraper and the walls is usually smaller (around $0.5 \mathrm{~mm}$ ) [22, 45]. Mechanical and shear forces are applied to the powder bed by the inner-piece against the walls of the rotating vessel creating shear zone that produces shear stress while the scraper removes the powder attached to the chamber wall. When the vessel rotates at a very high speed, the intense interaction results in the generation of significant amount of thermo-mechanical energy leading to the formation of a uniform layer of the guest material over the carrier [45]. 
The next set of developments were geared towards machines capable of generating high shear milling and grinding environment in order to break the agglomerates into primary fine powder followed by its deposition on to the carrier. An example is a device developed by the Japanese research group $\left(\right.$ Hybridizer $^{\circledR}$ ) which was based on providing very high shear impaction force that was proven to enhance the dissolution of low solubility drug $[20,21]$. The device was made up of high speed motor with a processing chamber containing six blades and powder recirculation circuit. The processing vessel was surrounded by a jacket with coolant to reduce the effect of heat generated during the process. The coating process was based on high impaction and dispersion forces as well as heat generated through friction from collisions of the powder mix with the baldes and the device walls [20, 26, 30, 33].

Both Mechanofusion ${ }^{\circledR}$ and Hybridizer $^{\circledR}$ promote chemical and physical guest-carrier interactions with chemical interaction being termed mechanochemistry [20, 23]. A typical example was the use of silica as a guest and cellulose as carrier which resulted in interaction between the silanol group $\mathrm{SiOH}$ of the silica and the hydroxyl groups on the cellulose forming - $\mathrm{O}-$ group that culminated in increase in hydrophobicity through release of a water molecule [23].

\subsection{Mild dry powder coating devices}

Several dry powder coating blenders were introduced that provided mild coating conditions. The Elliptical rotor mixer (Theta Composer ${ }^{\circledR}$ ) included an elliptical vessel with rotor operating in opposite directions. Powder coating occurred when the particles were subjected to high shear force and compression between the rotor and vessel wall during rotation [46].

Magnetically assisted impaction coating $\left(\mathrm{MAIC}^{\circledR}\right)$ is a device that provides mild coating when magnetic particles (made of barium ferrite and coated with polyurethane to minimise contamination) that are larger than the carrier are mixed with the powder mixture within a 
magnetic field resulting in collisions between components and the wall of the device [40]. The device is surrounded by an oscillating magnetic field that excites the magnetic particles resulting in high mobility inside the mixing chamber imparting energy on the guest and carrier particles causing collisions and impaction force on the guest and carrier particles thereby facilitating the coating process $[40,47]$. This method is best used when particle size exceeds $25 \mu \mathrm{m}[23,48]$. Rotating Fluidised Bed Coater $\left(\mathrm{RFBC}^{\circledR}\right)$ is based on centrifugal fluidization with the aid of radial flow of gas through a porous wall that results in mild coating process. Owing to the high rotations of the processing vessel, centrifugal and shear forces are applied to the powder bed with the aid of fluidising gas leading to increased collision between particles that facilitates de-agglomeration and subsequent coating [23, 40, 49].

Surface particle coating concurrently with particle micronisation using fluid energy mill $\left(\mathrm{FEM}^{\circledR}\right)$ was introduced and is best used for cohesive materials with particle size $<25 \mu \mathrm{m}[22$, 48, 50]. This process was used to produce nano-sized particles using high velocity gas producing a fluidised bed containing micron sized carrier particles. A premixed carrier /guest mixture is fed into a feed funnel supported by air inlet, and the high velocity gas introduced into the system provides fluidised bed that supports collision between particles providing strong shear force that results in micronisation of the carrier and guest particles with subsequent coating of the nano-sized guest particles on the surface of the carrier particle (micron size) [50]. Table-1 summarises the main dry powder coating devices with their key properties and limitations. 
Table 1 Main technologies used for producing functionalised particles through dry powder coating.

$\begin{array}{lll}\text { Instrument } & \text { Properties/ Advantages } & \text { Limitations } \\ \text { Mechanofusion } & \text { - High shear and compression forces. } & \text { - Heat generated, not suitable for thermo- labile material. } \\ & \text { - Speed 200-10,000 rpm. } & \text { - There is particle attrition or even fracture of carrier materials. } \\ & \text { - Temperature is generated resulting in fusion between guest and carrier. } & \text { Not suitable for unstable or fragile carrier material. Possible } \\ & \text { - Very strong bonding (physical and /or chemical). } & \text { transform to amorphous or different polymorphs } \\ & \text { - A GMP model for pharmaceutical applications is present with a capacity } & \text { - Batch type not ideal for continuous coating process. } \\ & \text { of 1L. } & \text { Possible change to the chemical and electronic status of the } \\ & & \text { components. }\end{array}$

Manufacturer/ Reference

Hosokawa- Japan

$[26,40]$

components.

\begin{tabular}{|c|c|c|c|}
\hline Hybridizer & $\begin{array}{l}\text { - High shear and compression forces. } \\
\text { - Very short processing time. } \\
\text { - Very fast rotating blades at a speed of 5,000-20,000 rpm. } \\
\text { - Powder recirculating unit that moves the particles in and out to ensure } \\
\text { homogeneity of the mix. } \\
\text { - Temperature builds up so fusion could happen. } \\
\text { - Produces chemical and physical interaction between host and guest. } \\
\text { - Can work with smaller carrier particles size }(0.1 \mu \mathrm{m}) \text {. }\end{array}$ & $\begin{array}{l}\text { - Heat generated not suitable for thermo- labile material. } \\
\text { - There is particle attrition or even fracture of carrier materials. } \\
\text { Not suitable for unstable or fragile carrier material. Possible } \\
\text { transform to amorphous or different polymorphs. } \\
\text { - Batch type not ideal for continuous coating process } \\
\text { Possible change to the chemical and electronic status of the } \\
\text { components }\end{array}$ & $\begin{array}{l}\text { Nara Machinery- Japan } \\
{[20,23]}\end{array}$ \\
\hline $\begin{array}{l}\text { Theta } \\
\text { composer } \\
\text { (Elliptical } \\
\text { rotor) }\end{array}$ & $\begin{array}{l}\text { - Softer coating. } \\
\text { - Shear stress and compaction force. } \\
\text { - Vessel speed 30rpm. } \\
\text { - Elliptical rotor speed 500-3000rpm. } \\
\text { - No major change in particle size or shape. } \\
\text { - Minimal heat generation, no thermal deterioration. } \\
\text { - Short processing time (2-10 minutes). }\end{array}$ & $\begin{array}{l}\text { - It is a batch type not ideal for continuous coating process. } \\
\text { - Less mechanical resistance of produced functionalised } \\
\text { particles compared with high force devices. }\end{array}$ & $\begin{array}{l}\text { Tokuju- Japan } \\
{[26,46]}\end{array}$ \\
\hline
\end{tabular}




\begin{tabular}{|c|c|c|c|}
\hline Instrument & Properties/ Advantages & Limitations & Manufacturer/ Reference \\
\hline $\begin{array}{l}\text { Magnetically } \\
\text { assisted } \\
\text { impaction } \\
\text { coating } \\
\text { (MAIC) }\end{array}$ & $\begin{array}{l}\text { - High shear force } \\
\text { - Soft coating with minimal change in particle shape or size. } \\
\text { - Insignificant amount of heat is generated (good for heat sensitive material). } \\
\text { - Use of magnetic field. } \\
\text { - Lower energy demand. }\end{array}$ & $\begin{array}{l}\text { - Due to the step of adding and removing the magnetic } \\
\text { particles there is a significant risk of contamination. } \\
\text { - It is a batch type not ideal for continuous coating process } \\
\text { (although there is a version of continuous process ) } \\
\text { - Less mechanical resistance of produced functionalised } \\
\text { particles compared with high force devices. }\end{array}$ & $\begin{array}{l}\text { Aveka- USA } \\
{[26,40]}\end{array}$ \\
\hline $\begin{array}{l}\text { Rotating } \\
\text { Fluidized Bed } \\
\text { Coater } \\
\text { (RFBC) }\end{array}$ & $\begin{array}{l}\text { - Soft coating. } \\
\text { - Centrifugal fluidization and shear forces are introduced. } \\
\text { - Can be used for very small host and guest particles } \\
\text { - Can be either batch or continuous type processing. }\end{array}$ & $\begin{array}{l}\text { - Less mechanical resistance of produced functionalised } \\
\text { particles compared with high force devices. } \\
\text { - Unwanted aerosolization of the fine guest particles. } \\
\text { - Limitations of the guest particle size (it should exceed the } \\
\text { mesh size of } 10 \mu \mathrm{m}) \text {. }\end{array}$ & $\begin{array}{l}\text { NJIT- USA } \\
{[23,40,49]}\end{array}$ \\
\hline $\begin{array}{l}\text { Fluid energy } \\
\text { mill (FEM) }\end{array}$ & $\begin{array}{l}\text { - Micronisation and coating. } \\
\text { - For nano and micron size guest and carrier respectively. } \\
\text { - Continuous process. } \\
\text { - Micronisation of the carrier particles resulted in an increase in magnitude } \\
\text { and heterogeneity in surface energy that can be quenched using guest } \\
\text { particles with low energy. }\end{array}$ & $\begin{array}{l}\text { - Less mechanical resistance of produced functionalised } \\
\text { particles compared with high force devices. } \\
\text { - Attrition of particles and possible transform to amorphous or } \\
\text { different polymorphs } \\
\text { - Pre-mixing of guest and carrier has to be done before their } \\
\text { addition to the FEM. } \\
\text { - There is a need to monitor various variables to ensure the } \\
\text { targeted functionalised particles are produced that include } \\
\text { feeding rate, feeding pressure and grinding pressure. }\end{array}$ & $\begin{array}{l}\text { Sturtevant Inc- USA } \\
{[50,51]}\end{array}$ \\
\hline
\end{tabular}




\section{Functionalities achieved using dry powder coating}

Owing to the advantages offered by the process, several applications of dry coating have been reported.

\subsection{Enhanced flowability}

Good flowability of dry powder is vital in various pharmaceutical operations like blending, die filling, tableting, capsule filling and re-suspending of powder in inhalers [36, 48, 52-54]. Enhancing the flow properties of API with size less than $50 \mu \mathrm{m}$ is one of the main challenges in formulation that is handled by the addition of flow aids or through processing such as wet and dry granulation. However, flow aids present challenges that lead to segregation and inconsistency of the powder bed. Additionally the glidants itself being cohesive is difficult to disperse with the cohesive powder using conventional blending devices. Granulation tends to jeopardise the surface area available for dissolution and requires multiple processing stages $[35,40,51]$. In direct compression, the presence of fine API with poor solubility and loading exceeding $10 \% \mathrm{w} / \mathrm{w}$ requires a strategy that enhances the flowability, maintains high surface area to aid dissolution and increases bulk density $[40,48,55]$. Dry powder coating has been used to produce functionalised blend with enhanced flowability by reducing the intrinsic cohesion of the fine powder [23, 29, 32, 35, 55-57]. Guest molecules coating on the surface of carrier particles is pursued as a spacer resulting in reduction of direct particle to particle contact and hence Van der Waals forces $[55,58]$. This phenomenon has been termed ballbearing or even lubricant effect [32]. Dutta and co-workers [59] reported that nano-additives may result in neutralising effect of the electrostatic charges on carrier particles. Alteration of surface roughness or asperities had an impact on cohesive properties of the powder. Addition of nano-sized guest particles on the surface of carrier using dry powder coating creates 
asperities on the surface and reduces the contact between particles resulting in an improvement of flowability $[32,56,60]$.

Dry powder coating was employed to produce nano-scale surface roughness of cohesive API that led to a reduction of cohesive force within the powder bed and enhancement of flowability [34, 48]. In an another study, titanium oxide was used as a nano sized guest particle $(15 \mathrm{~nm})$ to coat polymethylmethacrylate $(5 \mu \mathrm{m})$ using mechanofusion ${ }^{\circledR}$ that resulted in a remarkable reduction of the angle of repose [23]. Similarly, dry powder coating of the cohesive corn starch with silica (nano-scale) resulted in an enhancement of flowability [34].

In a recent study [51], results of simultaneous micronisation and surface dry coating of Ibuprofen powder using FEM resulted in enhancement of the flowability and stabilisation of the surface energy and a significant reduction of the van der Waals dispersive forces. Unlike the micronized uncoated ibuprofen that demonstrated an increase in surface energy, stabilisation and reduction in surface energy ("passivation") was achieved for the coated particles. The degree of surface area coverage of silica guest particles to enhance the flowability (spacing) produces asperities on the carrier particles which should not be less than $20 \%$ (preferably $40 \%$ ) whereas the optimal guest particle size to improve flowability ranges between $10-20 \mathrm{~nm}$. Besides, the surface area coverage should never exceed $100 \%$ $[32,51,60]$. Micronisation process results in an increase in surface energy which is attributed to exposure of the higher energy crystal planes upon micronisation [51]. Such an increase in surface energy demonstrated a linear relationship with particle size reduction. However, upon dry powder coating, high energy sites are quenched and then passivated due to the coverage of the guest particles leading to a reduction of the expected increase in cohesiveness of the micronized carrier particles [51]. 


\subsection{Improved fluidisation properties / aerosolization and dispersion}

There is a close association between flowability, dispersibility and aerosolization. Powder dispersion in gas (dry dispersion) involves breakdown of the agglomerates into their primary particles upon the application of force that is enough to overcome the strong interparticulate cohesive forces [52]. Besides, dispersing nano particles is even more challenging due to high cohesive force compared to individual particle weight and large surface area/volume ratio [17]. Application of dry powder coating of carrier particles with nano sized guest particles (preferably with low surface energy) will create asperities on the surface that improves the dispersion of the powder through the reduction of both surface energy and contact area [52]. The level of asperities could be controlled through the optimisation of surface coverage as well as guest particles (nano-size) [32]. APIs within Geldart group C were dry coated with a nano-sized guest particles resulted in an improvement of their fluidisation that enabled further wet coating process [60]. Leucine (a low surface energy amino acid) was used to coat the surface of potassium chloride $(\mathrm{KCl})$ using $\mathrm{FEM}^{\circledR}$ where simultaneous micronisation and dry coating occurred. $\mathrm{KCl}$ was micronized to reach $5 \mu \mathrm{m}$ while Leuicine particles were in the nano-size range [52]. Using pressure titration curves it was found that uncoated particles required more energy (dispersion pressure) to break the agglomerates contrary to dry coated powders that needed much lower energy for dispersion [52].

Moreover, the development of respiratory aerosols requires APIs with particle size not exceeding $5 \mu \mathrm{m}$ to ensure efficient transport to the target locations in the lung [29]. However, powders tend to be extremely cohesive resulting in poor flowability and aerosolization behaviour. Dry powder coating technique was used to produce a carrier free high dose dry powder inhalers with acceptable flowability, fluidisation as well as de-agglomeration properties [29]. Using model hydrophilic (salbutamol) and hydrophobic (salmetrol and 
traiamcinolone) carriers with different particle size and shape, functionalised particles were produced using mechanofusion ${ }^{\circledR} .5 \%$ magnesium stearate was used as guest. Results revealed no growth of particles, enhancement of flowability and effective aerosol delivery due to the reduction of the intrinsic powder cohesive forces [29]. Furthermore, mechanofusion ${ }^{\circledR}$ was utilised to fluidise micronized lactose particles that were coated with magnesium stearate as guest particles which resulted in an increase in the flow function coefficient (FFC) from very cohesive to easily flowing [57].

\subsection{Homogenous blend}

Several studies demonstrated the use of dry powder coating technique to promote content uniformity, where the fine API particles are consistently distributed over the carrier particles leading to a homogenous blend [20]. In order to ensure blend homogeneity using dry powder coating technique, the carrier material needs to be within a narrow particle size distribution to minimise segregation between coated and non-coated particles [23, 55]. Song and colleagues [11] revealed that interactive mixtures produces homogenous blends, nonetheless, changing process parameters as well as crystal form caused variation in content uniformity. The results demonstrated that deviations from content uniformity are attributed to the presence of agglomerates of non-coated particles. The study concluded that different crystal forms require different processing parameters as the affinity of the guest molecule to the carrier varied depending on crystal habit [11].

\subsection{Enhanced dissolution of poorly water soluble APIs}

Since the rate of absorption of poorly soluble drugs is often limited by the rate of dissolution, measures to enhance the dissolution rate of API have a major impact on its absorption [61]. Although reducing the particle size of API leads to increase in surface area and then a 
proportional increase in dissolution rate of poorly water soluble drugs, the powder bed of small particle size tends to be cohesive leading to manufacturing problems pertinent to low flowability and low bulk density due to cohesive powder remaining as agglomerates [48, 61]. The change in dissolution pattern is primarily dependant on the wettability and solubility of the carrier material [20]. The solubility of the excipient used as carrier can affect the dissolution rate with soluble carriers resulting in an enhancement in the dissolution profile of the drug whereas the less soluble carriers tend to slow the dissolution rate as the particles are attached to the carrier [62-65]. When preparing a formulation with a very potent or low load drug, the use of a triturating agent is a common practice (usually insoluble e.g., dibasic calcium phosphate), which will in turn reduce the dissolution rate of the drug [63]. To enhance dissolution using hybrid blending, the model is built on adsorption of the fine particles of hydrophobic drug on to the outer surface of water soluble large carrier particles. Once placed in dissolution medium, the carrier particles will dissolve in water leading to the dispersion of the drug particles through the aqueous media preventing hydrophobic particles from aggregating and therefore enhancing their dissolution. Various water soluble excipients like lactose, mannitol, sorbitol and sodium chloride were used in preparing the functionalised particles $[22,24,39,43,66]$. To reach an optimum dissolution of APIs, particles should be completely dispersed in the dissolution fluid [43]. However, upon increasing the concentration of the guest API to a limit that exceeds the surface coverage of the carrier excipient, the remaining API particles tends to agglomerate resulting in a reduction of the dissolution rate [66].

Various strategies were reported to enhance dissolution profile that included optimising guest (drug) concentration and particle size, addition of surfactant (at a micro level) (the addition of surfactant will break or prevent the agglomeration hence enhance dissolution) or the use of different type of excipients. Allahham and co-worker [66] modelled the dissolution data 
using a bi-exponential equation (one related to dissolution from aggregated particles and the second is from dispersed particles). Dissolution rate of Gliclazide, a poorly water soluble API, using interactive mixture was enhanced by using a water soluble carrier (lactose, mannitol, sorbitol, maltitol and $\mathrm{NaCl}$ ) [24]. Interactive mixtures were produced to increase the dissolution of sparingly soluble drugs by mixing them with a water soluble carrier where the dissolution rate increase is due to the increase in surface area and enhancement of the wettability [61, 67-70]. Similarly, the concept of interactive mixtures was employed to enhance the dissolution profile of nifedipine, a poorly-water soluble drugs by adsorbing it on to a water soluble carrier (polyethylene glycol 3350) [71].

Han and co-workers [50] investigated a dual process to enhance the dissolution of sparingly soluble class II drug like ibuprofen which included micronisation of the API followed by dry coating using $\mathrm{FEM}^{\circledR}$ to produce API particles dry coated with the nano-silica. Micronisation of the API increased the surface area and therefore accelerated dissolution while silica particles increased the surface roughness promoting flowability of the API. Similar to other findings, dissolution rate was higher when the guest material is hydrophilic (e.g., polyvinyl pyrrolidine) compared with hydrophobic guest particles such as silica. Nevertheless, the dissolution rate in both cases was higher than the micronized or unaltered API. Tablets compacted from the blend with high API loading (ibuprofen 30-70\%) resulted in dissolution profiles that produced $80 \%$ release in less than 5 minutes [48].

\subsection{Material Protection}

Few examples have been reported on using dry powder coating to produce functionalised particles that possess resistance against environmental conditions like oxygen, humidity and light [60]. Powdered magnesium was coated with silica and carnuba wax as guest particles to reduce the hygroscopic properties of magnesium [39]. 


\subsection{Other applications}

Several applications using the concept of dry powder coating were reported in the engineering and medical fields aimed at enhancing particle performance [42]. For example, fine aluminium particles were coated with organic or in-organic materials to prevent agglomeration and improve combustion behaviour [56]. The fracture toughness of an artificial bone forming material (hydroxyapatite) was enhanced by dry coating with zirconia. Furthermore, tiny glass bubbles were coated with titania for its unique application to manage oil spills. Dry coated particles carrying titania react with oil and decompose it while the glass bubbles allow for the floating of the material over water that eventually will be mixed with sand [23].

Kanda and colleagues [27] used dry coating to produce a novel formulation to quench bad body odour. Known materials (e.g., zinc oxide and aluminium chlorohydrate) that can be used to quench odours have limited applications due to their physical properties of clogging aerosols and rough texture. Functionalised particles made from spherical polyethylene powder (core material) with zinc oxide or aluminium chlorohydrate as guest particles resulted in an improvement in texture while retaining the odour quenching power. Similarly, Nanko and colleagues [42] used mechanofusion ${ }^{\circledR}$ to produce thermoelectric $\mathrm{Mg}_{2} \mathrm{Si}$. 


\section{Challenges}

Although dry powder coating is a one-step technique, there are several challenges that need to be addressed. The degree of surface coating and the homogeneity of the mixture is not only dependant on the materials used (both carrier and guest) but also on the type of instrument used $[33,39]$. Therefore, variability could be an issue between different types of blenders or even between different processing parameters $[19,20]$. Some of the dry coating technologies generate heat that may affect the stability of thermo labile actives [35, 72]. Furthermore, particle attrition and/or heat generated may result in polymorphic and amorphous transformations which may jeopardise stability or even safety of components [20, 22]. Technologies such as $\mathrm{MAIC}^{\circledR}$ present the risk of introducing contaminants owing to the addition and removal of magnetic particles [40,42]. Additionally, moisture content with in carrier and guest particles may affect the coating process. For instance, the increase in moisture content of potato starch from $1.2 \%$ to $14.3 \%$ increased dry coating efficiency of aspirin guest particles from $2.1 \%$ to $3.5 \%$ (when $5 \%$ aspirin was added to the mixture) [21] as this could be attributed to the increase in adhesive forces owing to higher surface tension of moisture. However, Saharan [22] also reported the effect of moisture content on either stabilising or destabilising the dry coated functionalised particles, where increasing the humidity above $40 \%$ resulted in a reduction in interaction between guest (sulphonamide) and the carrier.

\section{Conclusion}

The use of dry powder coating is a promising technique that has various applications in pharmaceutical drug delivery. Based on this review, dry particle coating has shown to be viable for production of functionalised particles with either new or improved characteristics. A range of devices are available for producing functionalised particles with variations in the 
performance depending on the force introduced into the system. It can be concluded that a wide range of novel pharmaceutical applications can be developed using the wide range of dry particle coating devices that are currently available.

\section{Expert Opinion}

Cohesive and insoluble APIs as well as excipients with poor flowability present significant processing, manufacture and clinical challenges within the pharmaceutical industry. Dry coating, where fine guest particles are adsorbed on to the surface of coarse carrier is a promising technique to overcome a wide range of pharmaceutical limitations through de agglomeration, enhancement of flowability, dose uniformity and reproducible manufacturing. It also provides additional advantages including cost effective, sustainable and environmental friendly processing conditions (requiring no solvents) and scalability.

The obvious tangible advantages through the enhancement of flowability, dissolution rate and blend homogeneity are a massive step forward in pharmaceutical research. Moreover, a lot of potential could be leveraged from this technique particularly in developing medicines for children and elderly, fixed dose combination products and sustained release formulations. This process could be part of the ever increasing demand in developing age appropriate formulations without the need for complicated multi stage processing that can increase the cost substantially and complicate manufacturability.

Potential within this domain can be accelerated with the development of devices that can overcome some of the challenges presented in this field. For instance, mechanofusion works on the principle of high centrifugal forces that generate heat thereby limiting its pharmaceutical application to heat labile materials. Similarly, processing materials using hybridiser can lead to particle attrition. Another aspect for consideration is the development of devices that can offer continuous processing as the current direction for growth within the pharmaceutical sector is geared towards developing equipment and processes that can offer 
continuous processing with inbuilt quality check points. The progress in this field, especially its translation within the pharmaceutical sector has been hampered by the lack of detailed studies and bench mark models that can offer sufficient knowledge base for pharmaceutical development. Perhaps the shift in focus within the material processing and the willingness to investigate and apply tools and techniques developed for applications within other domains such as mechanical/chemical engineering could possibly open avenues for not only interdisciplinary research but also for effective translation of technologies across the different sectors and expertise.

It is evident that greater focus and attention needs to be offered to dry particle coating as it presents solutions to a wide range of issues within the pharmaceutical industry. One of the distinct advantages of this technology is the cross application of the fundamental principles to develop solutions for a wide range of different problems. For instance understanding the role of surface texture of carrier particles on the strength of interaction between the guest and carrier particles can provide vital information on its impact on flowability, guest stability (as a hybrid particle) as well as functionality of the resultant particles. Similarly, an accurate and extensive demonstration of the requirements of each system to produce the targeted particles and also the lack of in-depth understanding of the mechanism needs to be explored. This current limitation also paves the way to evaluate the suitability of a wide range of analytical techniques to study particle characteristics both before and after composite blending. Surface characterisation techniques such as atomic force microscopy, surface interferometry and micro CT scanning should be investigated to generate a wider portfolio of material understanding which will form the basis for selection criteria for both processing as well for identifying their limitations in composite blend formation. 


\section{Declaration of interest}

The authors would like to acknowledge Aston University (Overseas bursary scholarship) for funding the PhD studentships for E. Dahmash. 


\section{Bibliography}

Papers of special note have been highlighted as either of interest $\left(^{*}\right)$ or of considerable interest $(* *)$ to readers.

1. Harnby N. An engineering view of pharmaceutical powder mixing. Pharm Sci Technol To 2000 9/1/;3(9):303-09.

2. Kaye BH. Powder mixing: London : Chapman.\& Hall., 1997.

3. Muzzio FJ, Robinson P, Wightman C, Brone D. Sampling practices in powder blending. Int J Pharm 1997;155(2):153-78.

4. Muzzio FJ, Goodridge CL, Alexander A, et al. Sampling and characterization of pharmaceutical powders and granular blends. Int J Pharm 2003;250(1):51-64.

5. Hancock BC, Garcia-Munoz S. How do formulation and process parameters impact blend and unit dose uniformity? Further analysis of the product quality research institute blend uniformity working group industry survey. J Pharm Sci 2012;102(3):982-86.

6. Paul EL, Antimo-Obeng V, A, Kresta SM, eds. Handbook of industrial mixing: science and practice Hoboken, N.J.: John Wiley and Sons-Interscience 2004.

7. Portillo P, M. Quality by design for continuous powder mixing. New Brunswick, New Jersey: The State University of New Jersey; 2008.

8. Portillo PM, Ierapetritou M, Tomassone S, et al. Quality by design methodology for development and scale-up of batch mixing processes. J Pharm Innov 2008;3(4):25870.

9. Deveswaran R, Bharath S, Basavaraj, BV, et al. Concepts and techniques of pharmaceutical powder mixing process: A current update. Research J Pharm Tech 2009;2(2):245-49.

10. Jallo LJ, Ghoroi C, Gurumurthy L, et al. Improvement of flow and bulk density of pharmaceutical powders using surface modification. Int J Pharm 2012;423(2):213-25.

11. Song M, De Villiers MM. Effect of a change in crystal polymorph on the degree of adhesion between micronized drug particles and large homogenous carrier particles during an interactive mixing process. Pharm Dev Technol 2004;9(4):387-98.

12. Bridgwater J. Invited review: Mixing of powders and granular materials by mechanical means-A perspective. Particuology 2012;10:397-427.

* This paepr highlights mixing mechanisms and provide the basis for cohesive powder behaviour. 
13. Alderborn G, Nyström C. Pharmaceutical powder compaction technology / edited by Gran Alderborn, Christer Nystrm: New York : Marcel Dekker, , 1996.

14. Aulton ME. Pharmaceutics : the science of dosage form design / edited by Michael E. Aulton: Edinburgh : Churchill Livingstone, 2nd ed., 2002.

15. Castellanos A. The relationship between attractive interparticle forces and bulk behaviour in dry and uncharged fine powders. Adv in Phys 2005;54(4):263-376.

* A good reference on powder classification and behaviour

16. Führer C. Interparticulate Attraction Mechanisms. In: Alderborn G, Nyström C, eds. Pharmaceutical Powder Compaction Technology. New York: Marcel Dekker, Inc 1995:1-15.

17. Shabanian J, Jafari R, Chaouki J. Fluidization of Ultrafine Powders. IRECHE- Rapid Communication 2012;4(1).

18. Orband JLR, Geldart D. Direct measurement of powder cohesion using a torsional device. Powder Technol 1997;92(1):25-33.

19. Honda H, Kimura M, Honda F, et al. Preparation of monolayer particle coated powder by the dry impact blending process utilizing mechanochemical treatment. Colloids and Surfaces A 1994;82(2):117-28.

(**) This article set the basis for dry coating /hybrid mixing and highlight initial attempts

20. Ishizaka T, Honda H, Kikuchi Y, et al. Preparation of drug-diluent hybrid powders by dry processing. J Pharm Pharmacol 1989;41(6):361-68.

21. Ishizaka $\mathrm{T}$, Honda $\mathrm{H}$, Ikawa $\mathrm{K}$, et al. Complexation of aspirin with potato starch and improvement of dissolution rate by dry mixing. Chem Pharm Bull 1988;36(7):256269.

22. Saharan VA, Kukkar V, Kataria M, et al. Ordered mixing: mechanism, process and applications in pharmaceutical formulations. Asian J Pharm Sci 2008;3:240-59.

23. Pfeffer R, Dave RN, Wei DG, Ramlakhan M. Synthesis of engineered particulates with tailored properties using dry particle coating. Powder Technol 2001;117(1-2):40-67.

(**) A good resource on dry coating process and technologies.

24. Saharan VA, Choudhury PK. Dissolution rate enhancement of gliclazide by ordered mixing. Acta pharmaceutica 2011;61(3):323-34.

25. Huo D, Zhang JS, Xu ZJ, et al. Synthesis of mixed conducting ceramic oxides $\mathrm{Sr} F e$ Co 0.5Oy powder by hybrid microwave heating. J Am Ceram Soc 2002;85(2):510-12.

26. Gera M, Saharan VA, Kataria M, Kukkar V. Mechanical methods for dry particle coating processes and their applications in drug delivery and development. Recent patents on drug delivery \& formulation 2010;4(1):58-81. 
27. Kanda F, Nakane T, Matsuoka M, Tomita K. Efficacy of novel hybrid powders to quench body malodors. J Soc Cosmet Chem 1990;41(3):197-207.

28. Gotō K, Masuda H, Higashitani K. Powder technology handbook: CRC Press, 1997.

29. Zhou QT, Qu L, Larson I, et al. Improving aerosolization of drug powders by reducing powder intrinsic cohesion via a mechanical dry coating approach. Indian J Pharm Sci 2010;394(1-2):50-59.

(*) Provide information about major application of dry coating

30. Alonso M, Alguacil F. Dry mixing and coating of powders. Rev Metalu 1999;35(5):315-28.

31. Alonso M, Satoh M, Miyanami K. The effect of random positioning on the packing of particles adhering to the surface of a central particle. Powder Technol 1990;62(1):3540 .

32. Chen Y, Yang J, Dave RN, Pfeffer R. Fluidization of coated group C powders. AIChE Journal 2007;54(1):104-21.

33. Alonso M, Satoh M, Miyanami K. Mechanism of the combined coatingmechanofusion processing of powders. Powder Technol 1989;59(1):45-52.

34. Yang J, Sliva A, Banerjee A, et al. Dry particle coating for improving the flowability of cohesive powders. Powder Technol 2005;158(1-3):21-33.

35. Zhou Q, Qu L, Larson I, et al. Effect of mechanical dry particle coating on the improvement of powder flowability for lactose monohydrate: A model cohesive pharmaceutical powder. Powder Technol 2011;207(1-3):414-21.

36. Zhou Q, Armstrong B, Larson I, et al. Effect of host particle size on the modification of powder flow behaviours for lactose monohydrate following dry coating. Dairy Sci Technol 2010;90(2-3):237-51.

37. Xie HY. The role of interparticle forces in the fluidization of fine particles. Powder Technol 1997;94(2):99-108.

38. Das SC, Stewart PJ. Characterising surface energy of pharmaceutical powders by inverse gas chromatography at finite dilution. J Pharm Pharmacol 2012;64(9):133748.

39. Ouabbas Y, Chamayou A, Galet L, et al. Effect of Mechanical dry Coating on the Flowability and the Wettability of Silica Gel Powder. Proceedings $\mathrm{XI}^{\circ}$ Congrès de la Société Française de Génie des Procédés Des réponses industrielles pour une société en mutation Saint Etienne, 9 au 11 octobre 2007, № 96, 2-910239-70-5 2007(96).

40. Beach L. Effect of dry particle coating on the properties of cohesive pharmaceutical powders. New Jersey: New Jersey Institute of Technology; 2011.

41. Smikalla M, Mescher A, Walzel P, Urbanetz NA. Impact of excipients on coating efficiency in dry powder coating. Int J Pharm 2011;405(1-2):122-31. 
42. Nanko $\mathbf{M}$, Abe $\mathrm{H}$, Takeda $\mathrm{M}$, et al. Fabrication of Thermoelectric $\mathrm{Mg} 2 \mathrm{Si}$ by Mechanofusion and Pulsed Electric Current Sintering. IOP Conference Series: Materials Science and Engineering; 2011: IOP Publishing; 2011. p. 012006.

43. Supabphol R, Stewart PJ. Aggregation during the dissolution of diazepam in interactive and granulated mixtures. Pharm Pharmacol Commun 1996;2(5):233-36.

44. Dubernet C, Benoit JP, Couarraze G, Duchene D. Microencapsulation of nitrofurantoin in poly(epsilon-caprolactone) - tableting and invitro release studies. Int J Pharma 1987;35(1-2):145-56.

45. Chen W, Dave RN, Pfeffer R, Walton O. Numerical simulation of Mechanofusion system. Powder Technol 2004;146(1):121-36.

46. Endoh S, Szepvolgyi J, Izumi K, et al. Experimental and theoretical analysis of mechanical coating process of particles with the theta composer. Chem Eng Commun 2004;191(10):1259-70.

47. Luczak A, Jallo LJ, Dave RN, Iqbal Z. Polymorph stabilization in processed acetaminophen powders. Powder technol 2013;236:52-62.

48. Han X, Ghoroi C, Dave R. Dry coating of micronized API powders for improved dissolution of directly compacted tablets with high drug loading. Int $\mathrm{J}$ Pharm 2013;442(1-2):74-85.

49. Quevedo J, Pfeffer R, Shen Y, et al. Fluidization of nanoagglomerates in a rotating fluidized bed. AIChE journal 2006;52(7):2401-12.

50. Han X, Ghoroi C, To D, et al. Simultaneous micronization and surface modification for improvement of flow and dissolution of drug particles. Int J Pharm 2011;415(12):185-95.

51. Han X, Jallo L, To D, et al. Passivation of high-surface-energy sites of milled ibuprofen crystals via dry coating for reduced cohesion and improved flowability. J Pharm Sci 2013;102(7):2282-96.

52. Ghoroi C, Han X, To D, et al. Dispersion of fine and ultrafine powders through surface modification and rapid expansion. Chem Eng Sci 2013:11.

53. Watano $\mathrm{S}$, Nakamura $\mathrm{H}$, Hamada $\mathrm{K}$, et al. Fine particle coating by a novel rotating fluidized bed coater. Powder Technol 2004;141(3):172-76.

54. Watano S, Imada Y, Hamada K, et al. Microgranulation of fine powders by a novel rotating fluidized bed granulator. Powder Technol 2003;131(2):250-55.

55. Mullarney MP, Beach LE, Davé RN, et al. Applying dry powder coatings to pharmaceutical powders using a comil for improving powder flow and bulk density. Powder Technol 2011;212(3):397-402.

56. Chen Y, Jallo L, Quintanilla MAS, Dave R. Characterization of particle and bulk level cohesion reduction of surface modified fine aluminum powders. Colloids and Surfaces A 2010;361(1-3):66-80. 
57. Zhou QT, Armstrong B, Larson I, et al. Understanding the influence of powder flowability, fluidization and de-agglomeration characteristics on the aerosolization of pharmaceutical model powders. Eur J Pharm Sci 2010;40(5):412-21.

58. Ramlakhan Mohan M. An experimental study of dry particle coating: Devices, operating parameters and applications. 2001.

59. Dutta A, Dullea L. A comparative evaluation of negatively and positively charged submicron particles as flow conditioners for a cohesive powder. AIChE Symp Ser; 1990;p. 26-40.

60. Chen Y, Yang J, Mujumdar A, Dave R. Fluidized bed film coating of cohesive Geldart group C powders. Powder Technol 2009;189(3):466-80.

61. Nystrom C, Westerberg M. The use of ordered mixtures for improving the dissolution rate of low solubility compounds. J Pharm Pharmacol 1986;38(3):161-65.

62. Stewart P, Alway B. Aggregation during the dissolution of diazepam in interactive mixtures. Particul Sci Technol 1995;13(3-4):213-26.

63. Du JP, Hoag SW. Characterization of excipient and tableting factors that influence folic acid dissolution, friability, and breaking strength of oil- and water-soluble multivitamin with minerals tablets. Drug Dev Ind Pharm 2003;29(10):1137-47.

64. Nagai Y, Yamamoto H, Takeuchi H. Improvement in dissolution property of poorly water-soluble drugs by using mechanofusion system. J Soc Powder Technol 2006;43(9):640.

65. Shaw LR, Irwin WJ, Grattan TJ, Conway BR. The effect of selected water-soluble excipients on the dissolution of paracetamol and ibuprofen. Drug Dev Ind Pharm 2005;31(6):515-25.

66. Allahham A, Stewart PJ. Enhancement of the dissolution of indomethacin in interactive mixtures using added fine lactose. European journal of pharmaceutics and biopharmaceutics : official journal of Arbeitsgemeinschaft fur Pharmazeutische Verfahrenstechnik eV 2007;67(3):732-42.

67. Westerberg M, Nystrom C. Physicochemical aspects of drug release .17. The effect of drug surface-area coverage to carrier materials on drug dissolution from ordered mixtures. Int J Pharm 1993;90(1):1-17.

68. Westerberg M, Nystrom C. Physicochemical aspects of drug release .12. The effect of some carrier particle properties and lubricant admixture on drug dissolution from tableted ordered mixtures. Int J Pharm 1991;69(2):129-41.

(**) This article provides useful insight about requiremnts for dry coating

69. Nilsson P, Westerberg M, Nystrom C. Physicochemical aspects of drug release .5. The importance of surface coverage and compaction on drug dissolution from ordered mixtures. Int J Pharm 1988;45(1-2):111-21. 
70. Westerberg M, Jonsson B, Nystrom C. Physicochemical aspects of drug release .4. The effect of carrier particle properties on the dissolution rate from ordered mixtures. Int J Pharm 1986;28(1):23-31.

71. Lee CC, Ong CL, Heng PW, et al. Interactive mixture as a rapid drug delivery system. Drug Dev Ind Pharm 2008;34(2):206-14.

72. Zhou QT, Qu L, Gengenbach T, et al. Investigation of the extent of surface coating via mechanofusion with varying additive levels and the influences on bulk powder flow properties. Int J Pharm 2011;413(1-2):36-43. 